\title{
Optical Network Net Present Value Optimization in Shared Path Protection Environments
}

\author{
Marc Ruiz, Luis Velasco, Jaume Comellas, Gabriel Junyent \\ Advanced Broadband Communications Center (CCABA) \\ Universitat Politècnica de Catalunya (UPC), Barcelona, Spain \\ E-mail: mruiz@ac.upc.edu
}

\begin{abstract}
Network operators are facing hard competition for opportunities in the telecommunications market, thus network investments must be carefully evaluated from the costs and revenues viewpoint before the decision making process. Since a great part of core network operators' revenues comes from the provisioned connectivity services, in this work we study the value expected to generate by optical networks when provisioning differentiated services in Shared Path Protection (SPP) environments. In this regard, two wavelength partitioning schemes are compared: the shared $(s h-W S)$ and the differentiated (diff-WS). The benefits of both schemes are evaluated from an economic perspective defining costs and revenues models and using Net Present Value (NPV). We define the Optical Network NPV Optimization (OVALO) problem as a NPV maximization problem. To solve OVALO, a linearized version of NPV is proposed as objective function of the Integer Linear Programming (ILP) model and an iterative algorithm is used to finally solve OVALO. The partitioning schemes are numerically compared on two network topologies. From the results, we show that $\operatorname{diff}$-WS maximizes resource utilization in the network without significantly increasing network costs, thus maximizing the network's value.
\end{abstract}

Keywords: Optical Networks, Resource Differentiation, Shared Path Protection, Net Present Value

\section{INTRODUCTION}

Competition among network operators to attract customers forces them to cut prices for the provided services at the risk of reducing turnovers if the amount of service does not rise. In consequence, costs must be carefully evaluated and kept under control. Total expenditures of a network operator can be separated into capital expenditures (CAPEX) and operational expenditures (OPEX). CAPEX is related with purchasing and installing fixed infrastructure such as buildings, equipments, cables, etc., whereas OPEX represents the costs of keeping the company operational and includes personnel costs, rented infrastructure, external services, etc. Some works have proposed methodologies and models for calculating OPEX and CAPEX costs in optical networks (e.g. see [1], [2]). Nevertheless, although costs can be used directly

This work has been partially founded by Spanish Science Ministry through TEC2008-02634 ENGINE project. Moreover, it has received funding from the European Community's Seventh Framework Programme FP7/2007-2013 under grant agreement $n^{\circ} 247674$ STRONGEST project. to compare among network alternatives, it is important consider also the generated revenues.

A great part of core network operators' revenues comes from the provisioned connectivity services. Being the core business, it is critical to perform a detailed analysis of the profitability of any investment project. In finance, the Net Present Value (NPV) is the most extended criterion to compare among investments [3]. NPV allows comparing long-term projects as it measures the generated cash flows in present value terms, thus relating revenues, OPEX, and CAPEX.

A good way to increase network operator's revenues is by meeting a wider range of client necessities using service differentiation. For instance, many companies do not consider the Internet access as a critical service. Therefore, the price they are willing to pay for it is generally low. On the contrary, business data traffic is usually associated with strict Service Level Agreements (SLAs), which makes this service significantly more expensive.

Typically, SLA contracts contain, among other parameters, the connection availability, that is, the probability that a connection will be operative at a random point in time. The way to improve connection availability in Dense Wavelength Division Multiplexing (DWDM) networks is by means of protection and restoration schemes. Particularly, Shared-Path Protection (SPP) has received much research attention, as it provides the best balance among availability, recovery time and resource utilization (e.g., see [4], [5]).

Essentially, SPP consists in providing two disjoint paths between source and destination nodes, where the working path is replaced by the backup path upon a failure affecting the former. The same backup resource (i.e., wavelength channel) may be shared so as to provide protection to multiple working paths, as long as they are mutually diverse. In fact, backup paths are configured to recover working paths from failures, but not used in normal conditions. Hence, this unused backup capacity can be used for supporting extratraffic, which would be preempted in case of working path failure.

In our previous work [6] we examined the provisioning of differentiated services in current Shared Path Protection (SPP) environments. To this end, two classes of service were 
defined: the SPP-based protected class (SP) and the best effort preemptable class (BE). This analysis revealed that current resource assignment policies are only able to provide a very poor grade of service to the supported BE traffic. Aiming at improving this performance, a novel resource partitioning scheme called diff-WS was proposed, which differentiates those wavelengths supporting each class of service in the network. Additionally, the revenues maximization problem was presented to design the optical network such that the operator's revenues were maximized. In the way to solve the design problem, we derived statistical models to obtain, given a certain grade of service, the highest traffic intensity for each class of service and resource partitioning scheme.

Nonetheless, in [6] we were solely concerned with revenues. In this paper we go a step further and define the OVALO problem as a DWDM network design problem to maximize optical network's NPV with revenues resulting from provisioning differentiated services. Since the optimal network topology to be used with each partitioning scheme might be different, and hence both costs and revenues, we use NPV to compare these schemes choosing the one providing the highest value.

The reminder of this paper is organized as follows. Section 2 briefly summarizes the considered wavelength partitioning schemes. Being the network traffic intensity a key issue to solve the problem, it also provides accurate statistical models to compute it for SP and $\mathrm{BE}$ traffic classes for both wavelength partitioning schemes. Section 3 states the OVALO problem, whose objective is to maximize NPV from deploying, operating, and serving differentiated traffic, while meeting certain Grade of Service (GoS) levels specified in terms of network blocking probability. To compute NPV for the partitioning schemes to be compared, we develop models for CAPEX (we assume that OPEX are proportional to CAPEX, so no OPEX model is developed in this work) and Revenues. In section 4 the OVALO ILP model and an iterative procedure to design the network for a given partitioning scheme are presented. The derived iterative algorithm and model are applied to different reference network topologies in section 5, illustrating that the expected NPV is maximized when applying the diff-WS scheme. Finally, section 6 concludes the paper.

\section{SUMMARY OF WAVELENGTH PARTITIONING SCHEMES AND INTENSITY MODELS}

For the sake of clarity, a summary of the wavelength partitioning schemes and intensity models proposed in our previous work [6] is reproduced in this section.

The topology of an optical network can be represented by a graph $G(N, E, W L)$, where $N$ represents the set of nodes, $E$ the set of links and $W L$ the set of available wavelengths in each link, with size $W$. Assuming that wavelength conversion is not available in the network we can split the graph $G$ into $W$ independent subgraphs $G^{i}(N, E, i)$, one per wavelength. Each subgraph $G^{i}$ represents the network connectivity through wavelength $i$.
As mentioned before, SPP achieves high resource efficiency by sharing the backup resources where possible. This resource sharing is attained by reserving the backup resources but postponing their allocation until the working path fails. SP connection requests are routed on subgraphs $G^{i}$, whereas BE connections are established using only resources reserved for the backup paths that are currently idle. Therefore, BE traffic is subordinated to the SP traffic. In other words, while the SP traffic blocking probability $\left(P b_{S P}\right)$ depends on the network topology $G$ and the offered load, that of the BE traffic $\left(P b_{B E}\right)$ also depends on the amount of resources loaned by the currently established SP connections. Furthermore, as BE connections are borne on reserved backup resources, they are torn down abruptly when the resources supporting them are unallocated, even if their requested holding time $(h t)$ has not expired yet. In this context, we defined the billable time $(b t)$ of a connection as the total time being operative. Thus, $b t=\rho \cdot h t$, where $\rho$ is the proportion of consumed $h t$ over the total. Note that $\rho_{B E} \leq 1$ as a consequence of the anticipated connection releases, whereas $\rho_{S P}=1$.

In view of the above, we proposed a novel wavelength partitioning scheme for provisioning differentiated traffic in DWDM transport networks. Our main objective was to improve the $\mathrm{BE}$ traffic performance and, as a result, the expected network revenues. Our proposal was to split the complete set of wavelengths into two different subsets of size $W / 2$, namely, $W L_{S P}$ and $W L_{B E}$, dedicated to SP working and backup path reservations, respectively. We called this wavelength partitioning scheme as differentiated wavelength set (diff-WS), in contrast to the traditional unpartitioned wavelength set where all resources are shared by working and backup paths $(s h-W S)$.

As was proven, the diff-WS approach provides higher GoS than the one provided by $s h-W S$ for BE traffic, which is achieved in certain network topologies at expenses of slightly lowering the amount of SP traffic served. Nonetheless, the total network revenues from both kinds of services are drastically leveraged when diff-WS was applied.

Regarding the traffic intensity, since we face dynamic traffic scenarios, four models were developed, one for each pair of class of service $j$ and wavelength partitioning scheme $k$. We concluded that a general parametrical formula with the number of links $(|E|)$ and the average path length in hops $(h)$ as independent variables could be used with specific exponents values, $\alpha, \beta$, and $\gamma$, for each $I_{j}^{k}$ model. Equation (1) shows the prediction model. Note that $I_{j}^{k}$ models the offered load per node belonging to the class of service $j$ and under the wavelength partitioning scheme $k$. In order to provide a confidence interval to the statistical models, the parameter $\varepsilon(k, j)$ collecting the relative error has been also included.

$$
I_{j}^{k}=\frac{10^{\alpha(k, j)} \cdot|E|^{\beta(k, j)}}{h^{\gamma(k, j)}} \pm \mathcal{E}(k, j) .
$$

Table 1 shows the values of the exponents and the relative error for each $I_{j}^{k}$ model. These parameters were obtained considering 16 wavelength channels per link. To illustrate the 
goodness-of-fit of the models, the values of the Pearson coefficient $\left(R^{2}\right)$ are also shown. As can be observed, all $R^{2}$ values are higher than $95 \%$, thus giving a tight fit for the offered load models.

TABLE 1. PARAMETERS AND OBSERVED AdJUST FOR THE INTENSITY MODELS

\begin{tabular}{|l|l|r|r|r|r|r|}
\hline Scheme $(k)$ & Class $(j)$ & $\alpha$ & $\beta$ & $\gamma$ & $\varepsilon(\%)$ & $R^{2}(\%)$ \\
\hline \multirow{3}{*}{ sh-WS } & SP & 0.222 & 0.931 & 3.07 & 5.62 & 99.2 \\
\cline { 2 - 7 } & BE & 1.522 & 0 & 6.40 & 9.94 & 95.1 \\
\hline \multirow{2}{*}{ diff-WS } & SP & 0.334 & 0.724 & 2.72 & 5.44 & 99.3 \\
\cline { 2 - 7 } & BE & 0.548 & 0.603 & 2.56 & 5.48 & 99.1 \\
\hline
\end{tabular}

In the reminder of this paper we use the $s h-W S$ and diff-WS wavelength partitioning schemes and intensity model to solve the OVALO problem.

\section{THE OVALO PROBLEM}

As stated in the introduction, connectivity services are in the core business of network operators and therefore it is critical to perform detailed analysis of the profitability of any investment project related with these services. In finance, the net present value (NPV) is the most extended criteria to compare among investments [3]. It can be computed as:

$$
N P V=\sum_{t=1}^{Y}\left[\frac{R E V E N U E S_{t}-O P E X_{t}}{(1+r)^{t}}\right]-C A P E X,
$$

where $Y$ is the total time period considered (in years), and $r$ is the annual discount rate.

\section{A. Revenues and costs models}

The revenues model consist on defining optical connections belonging to a class of service $j$, each one providing service levels specified by a SLA. One important aspect of an SLA is the availability of the connections. As was previously discussed, protection and/or restoration schemes can be implemented to increment the availability of the connections. In this paper, we assume that only two classes of service have been put on the market: the shared protected (SP) class which uses shared protection to provide a highly available service, and the best effort (BE) class which uses protecting resources to provide extra traffic while those resources are not being used (failure free conditions).

The revenues from selling these services can be computed knowing the billable time. We defined $b t_{j}^{k}$ as the billable time of service class $j$ during a certain time interval $\Delta t$ (e.g. one year) and using the wavelength partitioning scheme $k[6]$.

$$
b t_{j}^{k}=|N| \cdot I_{j}^{k} \cdot \rho_{j}^{k} \cdot\left(1-P b_{j}\right) \cdot \Delta t .
$$

Then, the revenues obtained from serving the classes of service over an optical network using the partitioning scheme $k$ can be computed as follows where $C_{j}$ stores the cost of one time unit of the class of service $j$.

$$
R E V E N U E S_{t}^{k}=\sum_{\forall j \in S}\left(|N| \cdot I_{j}^{k} \cdot \rho_{j}^{k} \cdot\left(1-P b_{j}\right) \cdot \Delta t \cdot C_{j}\right)
$$

Additionally, we assume traffic intensity being constant with time, and thus revenues are also constant in the period of time where the NPV is being considered.

Regarding CAPEX costs, they can be computed as the sum of the cost of the nodes plus the sum of the cost of the links. To model the cost of the optical nodes we assume that they are based on the design presented in [7] for colorless nodes, were the cost of increasing the nodal degree is lineal up to a maximum of 8 when 1x9 wavelength selective switches (WSS) are used. Then, the cost of an optical node can be modeled as the summation of three factors: firstly, $C_{B A S E}$ which includes the cost of common equipment (e.g., sub-rack, power cards, control card, etc.) and the installation cost; secondly, the cost of the WDM interfaces, which is proportional to the nodal degree $(\delta)$ and to the cost of one WDW interface, $C_{\text {Trunk }}$; finally, the cost of the access to the optical network, which is proportional to the cost of every optical transponder, $C_{\text {Transponder }}$, and depends on the traffic intensity offered to the network. To compute the number of transponders to install in every optical node we assume that the maximum of traffic doubles the mean. Then, the higher amount of connections having a given node as source or destination in the same time period can be computed as:

$$
\operatorname{maxPaths}^{k}=\left\lceil\sum_{\forall j \in S} 2 \cdot I_{j}^{k}\right\rceil .
$$

On the other hand, the cost of a link $e\left(C_{e}\right)$ includes: firstly, the cost of using a pair of optical fibers, which is proportional to the cost per km, $C_{F O K m}$, and to the length of the link, $L(e)$. It is worth noting that we consider that the optical cables are already deployed so the total deployment cost of an optical cable is allocated among the different networks using some fibers from it; secondly, the cost of the optical amplifiers and dispersion compensators, each with a cost $C_{O A}$, which number depends on the length of the link and can be computed as floor $(L(e) / A S)$, where $A S$ is the amplifier span length.

Finally, we can accurately estimate the CAPEX to deploy an optical network using the partitioning scheme $k$ as:

$$
\begin{aligned}
C A P E X^{k} & =\sum_{\forall n \in N}\left(C_{B A S E}+\delta_{n}^{k} \cdot C_{\text {Trunk }}+\left\lceil\sum_{\forall j \in S}\left(2 \cdot I_{j}^{k}\right)\right] \cdot C_{\text {Transponder }}\right) \\
& +\sum_{\forall \in \in E(k)}\left(L(e) \cdot C_{F O K m}+\left\lfloor\frac{L(e)}{A S}\right\rfloor \cdot C_{O A}\right)
\end{aligned}
$$

Regarding operational costs, several studies can be found in the literature providing models to compute OPEX only partially. Authors in [2] present an activity based approach to quantify the cost part of the operational processes, those related with repair and provisioning. Non event-driven processes, such as preventive maintenance, or other related with the technology used or the amount of served traffic, such as power consumption, are, however, omitted. A specific ILP model to design the network minimizing the energy consumption is presented in [9]. Finally the authors in [10] quantify the impact of the protection schemes and the network component's availability on the OPEX. 
Nevertheless, the OPEX is somehow related with the network's dimension, i.e. number and complexity of the nodes, total length of the links, etc., and then somehow related with CAPEX. Therefore and without loss of generality, in this paper we consider that OPEX is proportional to CAPEX, i.e., OPEX $=\eta^{*} \mathrm{CAPEX}$, where $\eta$ is in the range $[0-1]$

\section{B. OVALO problem statement}

From equation (2), it is clear that projects' value can be improved by increasing revenues or, alternatively, reducing costs. We propose the Optical Network Net Present Value Optimization (OVALO) problem to find the network design associated with a partitioning scheme from the presented in the previous section, which maximizes NPV. It is worth noting that real backbone optical transport network topologies (e.g. [8]) share planarity as a common characteristic. Moreover, to provide protection, two-connectivity is another requirement for feasible topologies.

Regarding traffic, we assume that the network must guarantee, as a result of some market research, a certain amount of SP traffic. Likewise, owing to its reduced price, we assume that the connectivity market demands a large amount of BE traffic.

Then, the OVALO problem can be formulated as follows: Given:

a) the physical topology of a network represented by a graph $G(N, E, W L)$,

b) a set $S$ of classes of service to be provided,

c) the network operator's pricing structure, specified by a fixed fee $C_{j}$ charged to the customers per time unit of class of service $j$,

d) the minimum SP intensity required $I_{S P}{ }^{\text {min }}$ and the blocking probability threshold $P b_{j}^{\max }$ allowed for every class of service $j$.

e) a set $K$ of wavelength partitioning schemes, index $k$. In this paper we assume $K=\{s h-W S$, diff-WS .

f) the network cost model,

g) the period of time where the study applies (e.g. 10 years) and the discount rate $r$.

\section{Output:}

a) a wavelength partitioning scheme $k$,

b) the set of links $E(k)$ in the network designed for the partitioning scheme $k$ ensuring that the resulting topology is planar and, at least, 2-connected,

c) the traffic intensities $I_{S P}{ }^{\max }$ and $I_{B E}{ }^{\max }$ for the given classes of service unleashing $P b_{j}^{\text {max }}$ thresholds.

Objective: Maximize the net present value for the designed network where the revenues come from serving traffic belonging to the defined classes. Thus, the OVALO objective function can be expressed as:

$$
\text { (OVALO) } \underset{\forall k \in K}{\operatorname{Maximize}} N P V^{k} \text {. }
$$

The next section presents models to solve the OVALO problem. Being its objective nonlinear, an iterative method that, at each iteration, solves a linearized version of the problem is proposed.

\section{OVALO OPTIMIZATION MODEL}

The model for the OVALO problem uses the following sets and parameters:

$\begin{array}{ll}N & \text { set of nodes, index } n \\ E & \text { set of links, index } e \\ C_{e} & \text { Cost of link } e \text { (as defined in section } 3) \\ \Omega(n) & \text { set of incident links on node } n \\ D & \text { set of different pairs source-destination }\left\{s_{d},\right. \\ & \left.t_{d}\right\}, \text { index } d \\ X & \text { set of exclusions to ensure planarity, index } x \\ \varphi_{e}{ }^{x} & 1 \text { if link } e \text { is in the exclusion } x, 0 \text { otherwise } \\ \delta m i n, \delta m a x & \text { min and max nodal degrees } \\ I_{S P}{ }^{\min } & \text { required traffic intensity for the SP class } \\ h_{\max } & h \text { threshold to guarantee } I_{S P}{ }^{\text {min }} \text {. It can be } \\ & \text { computed using eq. (1) } \\ a & \text { desired number of links } \\ M & \text { a large positive constant }\end{array}$

We use the set $D$ to ensure that two link-disjoint routes can be found in the designed network topology (2-connectivity condition). Note that if $D$ does not contain all node pairs, one (or more) 2-connected subgraph(s), containing the source/ destination nodes, will be obtained instead.

Additionally, the following variables are defined:

$\zeta_{e} \quad$ binary, 1 if link $e$ is in the designed network, 0 otherwise

$\omega_{e}{ }^{d} \quad$ binary, 1 if demand $d$ uses link $e$ for the primary route, 0 otherwise

$\kappa_{e}^{d} \quad$ binary, 1 if demand $d$ uses link $e$ for the secondary route, 0 otherwise

$\delta_{n} \quad$ stores the nodal degree of node $n$

Using this notation, introducing the intensity model from eq. (1) to the NPV expression in eq. (2), and adapting it to be used as the objective function of OVALO (i.e., removing constants, grouping terms, etc.), we can write that for a given partitioning scheme $k$ :

$$
\begin{aligned}
\operatorname{maximize} N P V^{k} \equiv & \varepsilon_{1}^{k} \cdot \sum_{\forall e \in E} C_{e} \cdot \zeta_{e}+ \\
& \varepsilon_{2}^{k} \cdot\left(\sum_{\forall d \in D} \sum_{\forall e \in E} \sigma_{e}^{d}\right)^{-\gamma(k, B E)}
\end{aligned}
$$


where:

$$
\begin{gathered}
\varepsilon_{1}^{k}=-1-\eta \cdot\left(\sum_{\forall t \in Y} \frac{1}{(1+r)^{t}}\right) \\
\varepsilon_{2}^{k}=10^{\alpha(k, B E)} \cdot|E|^{\beta(k, B E)} \cdot|D|^{\gamma(k, B E)} \cdot\left[\left(\sum_{\forall \in Y Y} \frac{1}{(1+r)^{t}}\right) \cdot\right. \\
\left.\cdot\left(|N| \cdot \rho_{B E}^{k} \cdot\left(1-P b_{B E}\right) \cdot \Delta t \cdot C_{B E}-2 \eta \cdot C_{\text {transp }}\right)-2 C_{\text {transp }}\right]
\end{gathered}
$$

In view of equation (8), we are facing to a non-linear problem. However, using mathematical regression techniques in the meaningful range of $h$ values, we found a model to linearize the nonlinear part of equation (8),

$$
\left(\sum_{\forall d \in D} \sum_{\forall \in E E} \varpi_{e}^{d}\right)^{-\gamma(k, B E)} \approx \varepsilon_{3}^{k} \cdot \sum_{\forall d \in D} \sum_{\forall e \in E} \varpi_{e}^{d}
$$

with:

$$
\varepsilon_{3}^{k}=-|D|^{\gamma(k, B E)-1} \cdot \tau^{k} \cdot\left(\frac{2 \cdot|E|}{|N|^{2}}\right)^{2 \gamma(k, B E)-1}
$$

where $\tau^{\text {diffWS }}=1.724$, and $\tau^{\text {shWS }}=2.933$.

TABLE 2. OVALO ITERATIVE MethoD

Input: $G(N, E, W L), X,\left\{L_{e}\right\}, I_{S P}^{\min }$, Costs and prices structures, $\delta \min , \delta \max , \alpha(k, j), \beta(k, j), \gamma(k, j), \varepsilon(k, j)$.

Output: $E(k)$, NPV

Compute $C_{e}$ for each link

Compute $D$

Compute $h_{\max }$

Set initial point for $a=a^{i n i}$

BestSolution. $N P V=$-Infinite

BestSolution. $E(k)=\Phi$

BestSolution. $a=0$

dir $=-1$

while true do

Solve OVALO ILP model with $a$ links

if feasible solution found then

Compute $N P V$

if $N P V<=$ BestSolution. $N P V$ then break

BestSolution. $E(k)=\left\{e \in E, \zeta_{e}=1\right\}$

BestSolution. $N P V=N P V$

BestSolution. $a=a$

else

$$
\begin{aligned}
& \text { if BestSolution. } a>0 \text { then } \\
& \text { break } \\
& \operatorname{dir}=+1
\end{aligned}
$$

end while

$$
a=a+d i r
$$

return BestSolution
An important conclusion from equations (10) and (12) is that the objective to maximize is a function of $-|E|^{\gamma(k, B E)}$ and, as a consequence, the number of links needs to be minimized since $\gamma(k, B E)$ is defined as strictly positive. In this regard, we propose an iterative method where at each iteration the problem is solved for a fixed number of links $a$. If the problem is feasible then $a$ is decremented and a new problem is solved. On the contrary, if the problem is infeasible, the last feasible solution must be the optimal solution. Table 2 shows the OVALO iterative method to obtain the optimal solution for a given partitioning scheme.

As shown in the algorithm, the number of iterations, and thus the time complexity, of the algorithm depends on selecting the initial value of $a$ as closer as possible to the optimal one. To this end, we propose to compute $a^{\text {ini }}$ by solving the simultaneous equations composed by equation (1) together with the expression in [11] to compute the average length of the shortest paths in a mesh network $(h \approx \operatorname{sqr}((|N|-$ $2) /(\delta-1)))$.

Finally, the OVALO ILP model for a fixed number of links can be formulated as follows:

(OVALO) maximize $\varepsilon_{1}^{k} \cdot \sum_{\forall e \in E} C_{e} \cdot \zeta_{e}+\varepsilon_{2}^{k} \cdot \varepsilon_{3}^{k} \cdot \sum_{\forall d \in D} \sum_{\forall e \in E} \varpi_{e}^{d}$

subject to:

$$
\begin{aligned}
& \sum_{\forall e \in \Omega(n)} \omega_{e}^{d}=1 \quad \forall d \in D \quad \forall n \in\left\{s_{d}, t_{d}\right\} \\
& \sum_{\forall e \in \Omega(n)} \omega_{e}^{d} \leq 2 \quad \forall d \in D \quad \forall n \in N-\left\{s_{d}, t_{d}\right\} \\
& \sum_{\substack{\forall e^{\prime} \in \Omega(n) \\
e^{\prime} \neq e}} \omega_{e^{\prime}}^{d} \geq \omega_{e}^{d} \quad \forall d \in D \quad \forall n \in N-\left\{s_{d}, t_{d}\right\} \quad \forall e \in \Omega(n) \\
& \sum_{\forall \in \in \Omega(n)} \kappa_{e}^{d}=1 \quad \forall d \in D \quad \forall n \in\left\{s_{d}, t_{d}\right\} \\
& \sum_{\forall e \in \Omega(n)} \kappa_{e}^{d} \leq 2 \quad \forall d \in D \quad \forall n \in N-\left\{s_{d}, t_{d}\right\} \\
& \sum_{\forall e^{\prime} \in \Omega(n)} \kappa_{e^{\prime}}^{d} \geq \kappa_{e}^{d} \quad \forall d \in D \quad \forall n \in N-\left\{s_{d}, t_{d}\right\} \quad \forall e \in \Omega(n) \\
& \omega_{e}^{d}+\kappa_{e}^{d} \leq 1 \quad \forall d \in D \quad \forall e \in E \\
& \sum_{\forall e \in E} \omega_{e}^{d} \leq \sum_{\forall e \in E} \kappa_{e}^{d} \quad \forall d \in D \\
& \sum_{\forall d \in D} \sum_{\forall e \in E} \omega_{e}^{d} \leq|D| \cdot h_{\max } \\
& \sum_{\forall d \in D}\left(\omega_{e}^{d}+\kappa_{e}^{d}\right) \leq M \cdot \zeta_{e} \quad \forall e \in E \\
& \sum_{\forall e \in E} \zeta_{e}=a \\
& \sum_{\forall e \in E} \zeta_{e} \cdot \varphi_{e}^{x} \leq 1 \quad \forall x \in X
\end{aligned}
$$




$$
\begin{gathered}
\sum_{\forall e \in \omega(n)} \zeta_{e}=\delta_{n} \quad \forall n \in N \\
\delta_{\text {min }} \leq \delta_{n} \leq \delta_{\text {max }} \quad \forall n \in N
\end{gathered}
$$

In the model, the objective function (13) indirectly maximizes NPV by maximizing its linearized and adapted approach. Constraints (14)-(20) ensure that the designed topology is 2-connected, i.e., a pair of disjoint routes (named primary and secondary) must exist in the network for every source-destination pair. Constraint (21) forces the primary route to be the shortest route. Constraint (22) ensures that the required traffic intensity for the SP traffic, $I_{S P}{ }^{\min }$, can be provided. It is worth noting that, traffic is not actually routed since it is assumed dynamic. Instead, eq. (1) is used to ensure that enough resources are placed in the network such that the required traffic intensity can be provided. Note that $h_{\max }$ can be computed using eq. (1) from $I_{S P}^{\min }$ and a number of links equal to $a$. Constraint (23) stores the use of every link. Constraint (24) guarantees that the desired number of links $a$ is provided. Constraint (25) ensures that the topology is planar. Finally, constraints (26) and (27) limit nodal degrees.

\section{ILLUSTRATIVE NUMERICAL RESULTS}

For evaluation, two backbone optical network series were created, starting from two well-known base topologies and adding extra connectivity. To this end, we used the moderately meshed 14-Node Deutsche Telecom (DT) and a quite sparse 20-Node NSFNET topology. Fig. 1 shows the resulting test topologies and reviews their most relevant characteristics.

The OVALO iterative method and model were implemented in iLog-OPL and solved by the CPLEX v.12.0 solver on $2.4 \mathrm{GHz}$ Quad-Core machines with $8 \mathrm{~GB}$ RAM.

Aiming at obtaining realistic CAPEX and revenues values, we have adapted equipment costs from [2], the price of

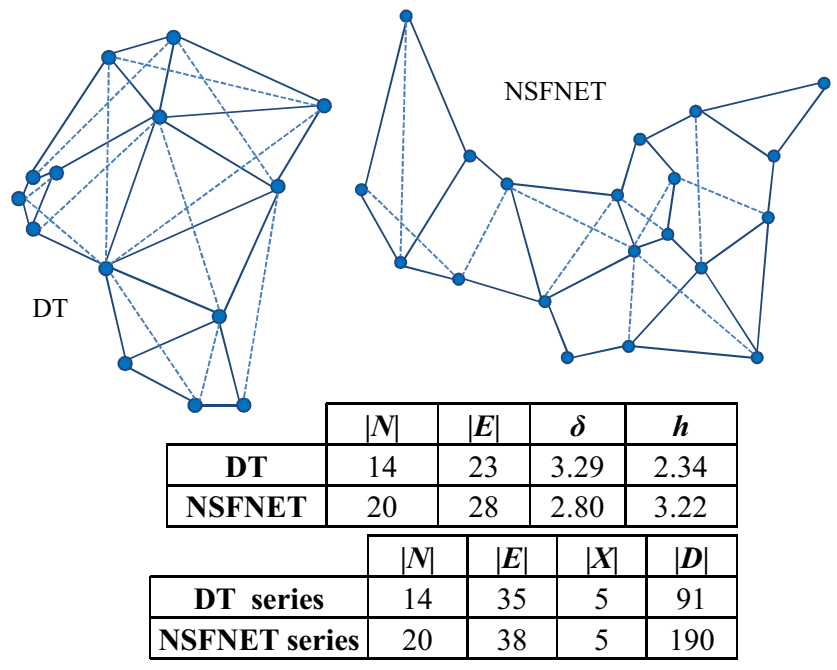

Fig. 1. Network topologies used for evaluation with their most relevant characteristics. Solid lines represent topology's original links whereas dotted lines are used for the added connectivity. connectivity services from [12], and the price ratio $C_{S P}: C_{B E}$ equal to $5: 1$ as concluded in [6]. Finally, we consider an amortizable period $Y=10$ years and a discount rate $r=5 \%$.

Before comparing results in terms of NPV, let us firstly to analyze the CAPEX and revenues values obtained after solving OVALO. To this end, Fig. 2 plots CAPEX as a function of $I_{S P}^{\min }$. It is worth noticing that the higher the required intensity the larger the amount of network equipment is needed to satisfy the required SP traffic, which is clearly appreciated for both network topologies. Although the CAPEX values are very close for low $I_{S P}$ intensities, for medium and high loads, networks for the sh-WS scheme are slightly cheaper ( $9 \%$ in the best case) that those for the diff$W S$ one. An interesting remark regarding the results for the NSFNET series is that the higher intensity with a feasible network solution is $I_{S P}{ }^{m i n}=1.5$ in contrast to 4.5 for the DT series. This is a result of the highest nodal degree for any feasible network topology: for the NSFNET series is 3.3 whereas for the DT series is 4.28.

We performed a similar study to compare revenues. Fig. 3 plots the values obtained for different $I_{S P}{ }^{\min }$ values. In this case, diff-WS provides higher revenues than $s h-W S$ for both series of topologies (up to $43 \%$ additional revenues). Note that revenues are computed from $I_{S P}{ }^{\min }$ and $I_{B E}{ }^{\max }$, therefore, since $I_{S P}{ }^{\min }$ is the same for both partitioning schemes, the difference lies in the amount of extra-traffic that can be supported in both partitioning schemes. In this regard, Fig. 3 also shows the $I_{B E}{ }^{\max }$ traffic that can be carried over the obtained network. As can be observed, diff-WS allows carrying several times more $I_{B E}^{\max }$ than $s h$-WS (up to 24 times for DT series and 38 for NSFNET series).

As a conclusion of Fig. 2 and Fig. 3, the diff-WS scheme provides higher revenues that $s h-W S$ but at the cost of more expensive networks. Therefore, it is useful to use NPV to compare between them. Fig. 4 plots the NPV values obtained for both partitioning schemes. As shown, diff-WS provides higher NPV values than $s h$-WS. In fact, the on average increment is closed to $30 \%$ for DT series and $60 \%$ for NSFNET series. Moreover, it is worth noting the strong linear correlation between NPV and $I_{S P}^{\min }$, in line with the trends observed in Fig. 2 and Fig. 3 for CAPEX and revenues, respectively.

Recall that OVALO looks for networks ensuring a given SP intensity $I_{S P}{ }^{\min }$. However, the resultant networks will be able to supporting higher intensity $\left(I_{S P}{ }^{\max }\right)$ if required, without increasing CAPEX. Then, a sensitivity analysis in terms of NPV can be done, where the revenues increase as result of providing $I_{S P}{ }^{\max }$. Table 3 shows, for each $I_{S P}{ }^{m i n}$ reference value, both the $I_{S P}{ }^{\max }$ values and the NPV increment (incNPV) that would be obtained provided $I_{S P}{ }^{\max }$ was served. The NPV gain using diff-WS instead of $s h-W S(\triangle N P V)$ is also detailed for $I_{S P}{ }^{\min }$ and $I_{S P}{ }^{\max }$. Note that NPV could increase up to $38 \%$ if SP traffic increases to $I_{S P}{ }^{\max }$. Although this increase would be slightly higher when $s h-W S$ is applied than when diff-WS is applied, the latter partitioning scheme would still remain providing the highest NPV values ( $23 \%$ of NPV gain). 

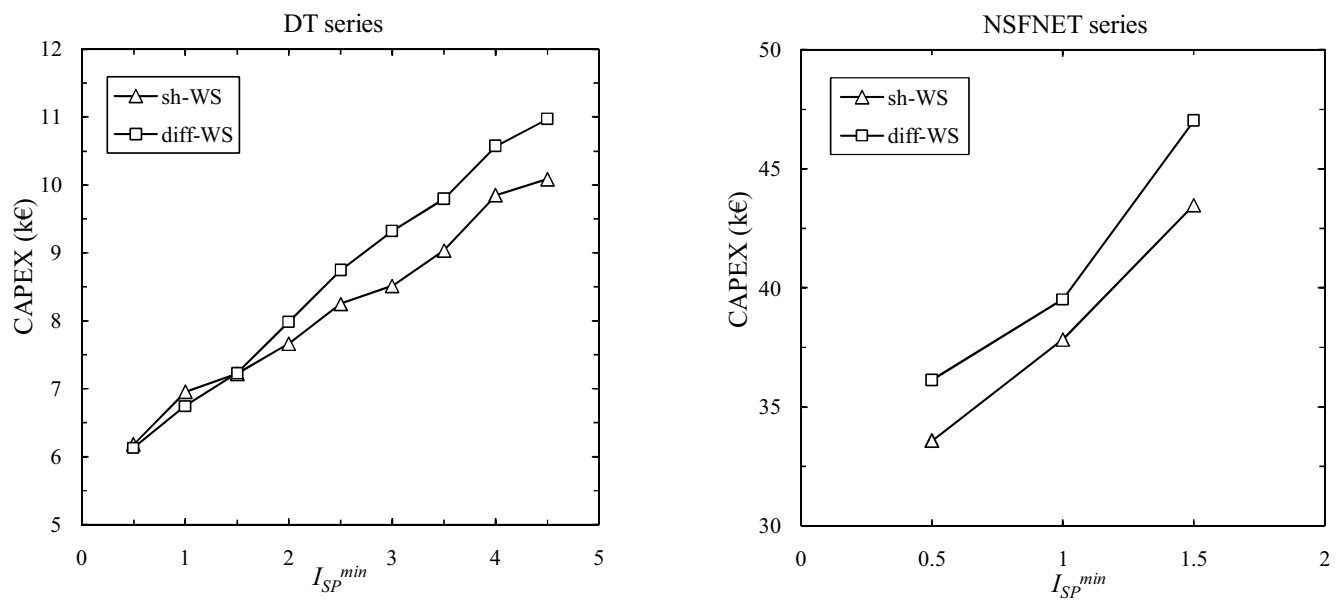

Fig. 2. CAPEX against the required SP intensity for the DT series (left) and NSFNET series (right).
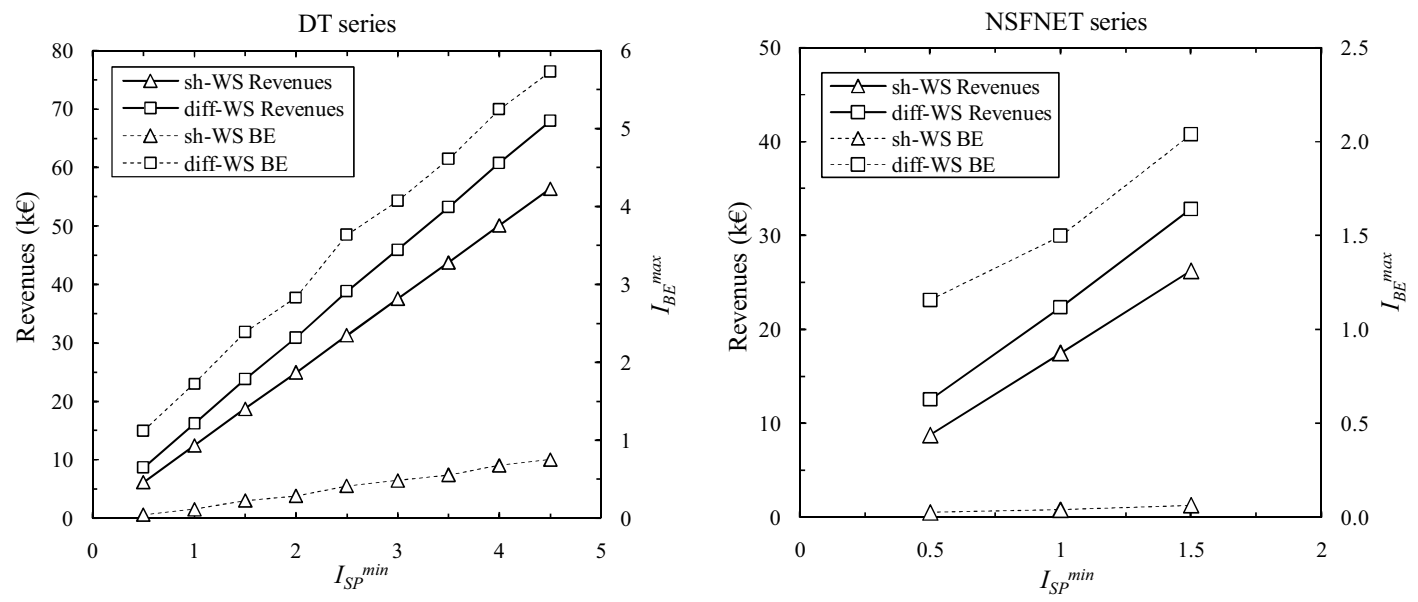

Fig. 3. Revenues (solid lines) and maximum BE intensity (dotted lines) against the required SP intensity for DT series (left) and NSFNET series (right).
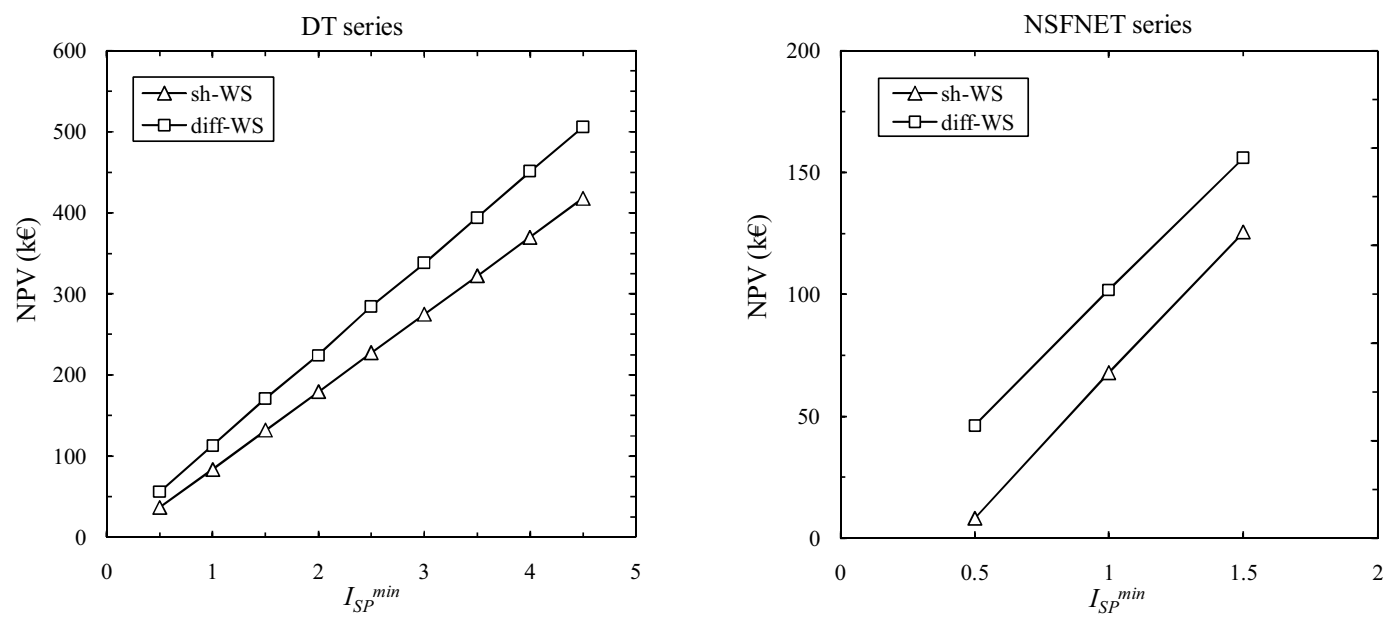

Fig. 4. Net Present Value against the required SP intensity for DT series (left) and NSFNET series (right). 
TABLE 3. NPV SENSITIVITY ANALYSIS FOR THE DT NETWORK TOPOLOGY

\begin{tabular}{|c|c|c|c|c|c|c|}
\hline & \multicolumn{2}{|c|}{$s h-W S$} & \multicolumn{2}{|c|}{$\operatorname{diff-WS}$} & \multirow{2}{*}{$\begin{array}{c}\Delta N P V \\
\left(I_{S P}{ }^{\text {min }}\right)\end{array}$} & \multirow{2}{*}{$\begin{array}{c}\Delta N P V \\
\left(I_{S P}{ }^{\max }\right)\end{array}$} \\
\hline$I_{S P}{ }^{\min }$ & $I_{S P}{ }^{\max }$ & incNPV & $I_{S P}{ }^{\max }$ & incNPV & & \\
\hline 0.5 & 0.73 & $37.46 \%$ & 0.80 & $33.20 \%$ & $53.02 \%$ & $43.26 \%$ \\
\hline 1.0 & 1.25 & $21.77 \%$ & 1.27 & $18.19 \%$ & $35.19 \%$ & $29.27 \%$ \\
\hline 1.5 & 1.86 & $25.53 \%$ & 1.80 & $13.96 \%$ & $29.46 \%$ & $19.87 \%$ \\
\hline 2.0 & 2.21 & $9.96 \%$ & 2.17 & $6.45 \%$ & $25.28 \%$ & $20.58 \%$ \\
\hline 2.5 & 2.80 & $11.11 \%$ & 2.85 & $10.28 \%$ & $25.14 \%$ & $23.97 \%$ \\
\hline 3.0 & 3.19 & $6.08 \%$ & 3.24 & $6.16 \%$ & $22.95 \%$ & $23.06 \%$ \\
\hline 3.5 & 3.73 & $6.20 \%$ & 3.72 & $4.95 \%$ & $22.23 \%$ & $20.62 \%$ \\
\hline 4.0 & 4.65 & $14.19 \%$ & 4.31 & $6.03 \%$ & $21.88 \%$ & $11.29 \%$ \\
\hline 4.5 & 5.09 & $11.65 \%$ & 4.76 & $4.59 \%$ & $21.06 \%$ & $12.11 \%$ \\
\hline & & & & Average & $28.34 \%$ & $23.02 \%$ \\
\hline
\end{tabular}

\section{CONCLUSIONS}

This paper has studied the economic impact of deploying and operating optical networks, from the investment project perspective. We considered differentiated service provisioning in SPP environments, under different resource differentiation schemes. More specifically, two wavelength partitioning schemes were compared: the diff-WS and the $s h$ $W S$. In our previous work we proved that networks designed with diff-WS allows obtaining higher revenues than those with $s h-W S$, due to a better BE traffic provisioning. However, no trade-off between revenues and cost was done to design the network.

In order to consider revenues and costs in the same model, the OVALO problem was defined as the problem of designing an optical network maximizing the expected NPV. Given a required SP intensity, OVALO finds the topology and the partitioning scheme that maximizes the NPV. Being NPV a non-linear function, linear programming techniques cannot be applied to obtain optimal solutions. Aiming to obtain exact solutions, an iterative method based on an ILP was proposed. A linear approximation of NPV was used as the objective function of that ILP. After solving the problem for different number of network links, the solution with the highest NPV is returned as the optimal one.

OVALO was solved for two well-known topologies enlarged with additional optical connectivity. From the results, we observed that NPV is maximized when diff-WS is applied. Although networks were slightly cheaper in terms of CAPEX under the $s h$-WS scheme, higher revenues coming from serving more $\mathrm{BE}$ traffic were expected for diff-WS. Moreover, the required $I_{S P}{ }^{\text {min }}$ could be increased to a maximum value $I_{S P}{ }^{\max }$ without increasing network costs. In that case, NPV could be increased up to $38 \%$ under the deployed infrastructure.

In light of the extensive evaluation, we are able to conclude that diff-WS maximizes NPV from operating a large range of different backbone networks.

\section{REFERENCES}

[1] R. Huelsermann, M. Gunkel, C. Meusburger, and D. Schupke, "Cost modeling and evaluation of capital expenditures in optical multilayer networks," OSA J. Opt. Netw., vol. 7, pp. 814-833, 2008.

[2] S. Verbrugge, et al., "Methodology and input availability parameters for calculating OpEx and CapEx costs for realistic network scenarios," OSA J. Opt. Netw., vol. 5, pp. 509-520, 2006.

[3] S. Ross, R. Westerfield, J. Jaffe, "Corporate Finance," McGraw-Hill, 2002.

[4] C. Ou, J. Zhang, L. H. Sahasrabuddhe, and B. Mukherjee, "New and improved approaches for shared-path protection in WDM mesh networks," IEEE/OSA J. Lightwave Technol., vol. 22, pp. 1223-1232, 2004.

[5] R. Muñoz, R. Casellas, R. Martinez, "An Experimental Signalling Enhancement to Efficiently Encompass WCC and Backup Sharing in GMPLS-enabled Wavelength-Routed Networks," In Proc. IEEE ICC, pp.5401-5406, 2008.

[6] L. Velasco, M. Ruiz, J. Perelló, S. Spadaro, and J. Comellas, "Service and Resource Differentiation to Maximize Network Operator's Revenues", IEEE/OSA Journal of Optical Communications and Networking, 2011.

[7] P. Roorda, B. Collings, "Evolution to Colorless and Directionless ROADM Architectures," National Fiber Optic Engineers Conference (NFOEC), 2008.

[8] C. Pavan, R. Morais, J. Ferreira, and A. Pinto, "Generating Realistic Optical Transport Network Topologies," IEEE/OSA Journal of Optical Communications and Networking, vol. 2, pp. 80-90, 2010.

[9] G. Shen and R. Tucker, "Energy-Minimized Design for IP Over WDM Networks," IEEE/OSA Journal of Optical Communications and Networking, vol. 1, pp. 176-186, 2009.

[10] C. Mas, O. Moe, and M. Jäger, "Impact of protection schemes and network component's availability on operational expenditures," OSA J. Opt. Netw. vol. 7, pp. 142-150 2008.

[11] S. Korotky, "Network Global Expectation Model: A Statistical Formalism for Quickly Quantifying Network Needs and Costs," IEEE/OSA J. Lightwave Technol., vol. 22, pp. 703-722, 2004.

[12] M. Xia, M. Tornatore, C. Martel, and B. Mukherjee, "Service-centric provisioning in WDM backbone networks for the future internet," IEEE/OSA J. Lightwave Technol., vol. 27, pp. 1856-1865, 2009. 